\title{
Lynfocytic colitis (Clinical case)
}

\author{
Colite linfocítica (Caso clínico)
}

Bruno Sousa ${ }^{(1,2,3)}$, Nelson Tavares ${ }^{(1,2)}$

${ }^{1}$ School of Sciences and Health Technologies, Universidade Lusófona de Humanidades e Tecnologias, Lisboa, Portugal

${ }^{2} \mathrm{CBIOS}$ - Research Center for Biosciences and Health Technologies

${ }^{3}$ Health Service of Autonomous Region of Madeira

e-mail: bruno.sousa@mail.com

Received /Recebido: 13/03/2018 Accepted / Aceite: 20/03/2018

Electronic Edition: www.alies.pt

A 67-year-old woman, retired, with several associated pathologies, presented at a Nutrition Consultation with complaints of diarrhea and abdominal cramps for approximately five months, medicated during this time by her General Practitioner with Racecadotril and Atyflor ${ }^{\circledR}$. Awaits consultation of gastroenterology.

Anthropometric evaluation

Weight: $97.6 \mathrm{~kg}$

Height: $164 \mathrm{~cm}$

BMI: $36.3 \mathrm{~kg} / \mathrm{m} 2$

Waist circumference: $117 \mathrm{~cm}$

Assessment of body composition (TANITA TBF $300 \circledR$ ) Body fat: $44.6 \%$

Fatty mass: $43.5 \mathrm{~kg}$

Fat free mass: $54.1 \mathrm{~kg}$

Total body water: $39.6 \mathrm{~kg}$

Analytical Parameters:

Hematology

Leukocytes: 9.8 x 103 / L (4.5 - 110)

Erythrocytes: 4.01 x 10-6 / L (3.80 - 5.80)

Hemoglobin: $12.3 \mathrm{~g} / \mathrm{dL}(11.5$ - 16.0)

Hematocrit: $37.2 \%$ (37.0 - 47.0)

Sedimentation rate: $39 \mathrm{~mm}(<30)$

Glycated hemoglobin (A1C) - 5.9\% (4.0 - 6.0)

Biochemistry

Glucose: $119 \mathrm{mg} / \mathrm{dL}(82.0$ - 115.0)

Urea: $35.1 \mathrm{mg} / \mathrm{dL}(8.0$ - 50.0)

Creatinine: $1.12 \mathrm{mg} / \mathrm{dL}(0.70-1.20)$

Uric acid $8.4 \mathrm{mg} / \mathrm{dL}(2.6-8.0)$

Total cholesterol: $196 \mathrm{mg} / \mathrm{dL}(<200.0)$

HDL cholesterol: $71.0 \mathrm{mg} / \mathrm{dL}(40.0$ - 60.0)

LDL cholesterol: $104.2 \mathrm{mg} / \mathrm{dL}(<175.0)$

Triglycerides: $104.0 \mathrm{mg} / \mathrm{dL}(<150.0)$
Mulher de 67 anos, reformada, com várias patologias associadas, apresenta-se na Consulta de Nutrição com queixas de diarreia e cólicas abdominais há cerca de cinco meses, entretanto medicada pela médica de família com Racecadotril e Atyflor ${ }^{\circledR}$. Aguarda consulta de gastrenterologia.

Avaliação antropométrica

Peso: $97,6 \mathrm{~kg}$

Estatura: $164 \mathrm{~cm}$

IMC: $36,3 \mathrm{~kg} / \mathrm{m} 2$

Perímetro da cintura: $117 \mathrm{~cm}$

Avaliação da composição corporal (TANITA TBF $300 \AA)$

Gordura corporal: $44,6 \%$

Massa gorda: $43,5 \mathrm{~kg}$

Massa isenta de gordura: $54,1 \mathrm{~kg}$

Água corporal total: $39,6 \mathrm{~kg}$

Parâmetros analíticos:

Hematologia

Leucócitos:9,8 10^3/ $\mu \mathrm{L}(4,5-110)$

Eritrócitos: 4,01 10^6/ $\mu \mathrm{L}(3,80-5,80)$

Hemoglobina: $12,3 \mathrm{~g} / \mathrm{dL}(11,5-16,0)$

Hematócrito: $37,2 \%(37,0-47,0)$

Velocidade de sedimentação: $39 \mathrm{~mm}(<30)$

Hemoglobina glicada (A1C) - 5,9\% $(4,0-6,0)$

Bioquímica

Glicose: $119 \mathrm{mg} / \mathrm{dL}(82,0-115,0)$

Ureia: $35,1 \mathrm{mg} / \mathrm{dL}(8,0-50,0)$

Creatinina: $1,12 \mathrm{mg} / \mathrm{dL}(0,70-1,20)$

Ácido úrico $8,4 \mathrm{mg} / \mathrm{dL}(2,6-8,0)$

Colesterol total: $196 \mathrm{mg} / \mathrm{dL}(<200,0)$

Colesterol HDL: $71,0 \mathrm{mg} / \mathrm{dL}(40,0-60,0)$

Colesterol LDL: $104,2 \mathrm{md} / \mathrm{dL}(<175,0)$ 
Alanine Aminotransferase: $26.4 \mathrm{U} / \mathrm{L}$ (14.0 - 54.0)

Aspartate Aminotransferase: $25.2 \mathrm{U} / \mathrm{L}(10.0$ - 35.0)

Gamaglutamyltransferase: 41.0 U / L (7.0 - 50.0)

C-reactive protein: $17.14 \mathrm{mg} / \mathrm{L}(<6.10)$

Hormonology

Free T4: $1.4 \mathrm{ng} / \mathrm{dL}(30.0$ - 260)

TSH: $5.32 \mathrm{IU} / \mathrm{mL}(0.30$ - 4.70)

Immunology

IgA (serum): $494.0 \mathrm{mg} / \mathrm{dL}$ (70.0 - 400.0)

Rheumatoid factor: $<12.5 \mathrm{IU} / \mathrm{ml}(<16.0)$

Anti-Nuclear Antibodies: negative

B.C. Anti-Cytoplasm Neutrophil, IgG: negative

B.C. Anti-CCP, IgG: $0.0 \mathrm{UA} / \mathrm{mL}(<5.0)$

B.C. Tissue Anti-Transglutaminase, IgA: $2.2 \mathrm{U} / \mathrm{mL}$ (negative: $<10$ )

Urine

Creatinine: $93.0 \mathrm{mg} / \mathrm{dL}(30.0$ - 1.7)

Microalbuminuria: $<0.7 \mathrm{mg} / \mathrm{dL}(<2.0)$

Clinical evaluation:

Background:

- Obesity II

- Depressive Syndrome

- Arterial hypertension

- $\quad$ Cardiac valvular disease - mild sclerosis of aortic valve; mild left ventricular hypertrophy; mild mitral and tricuspid regurgitation

- $\quad$ Diabetes mellitus type 2

- Fibromioma of the uterus

- Fibromyalgia

- Obstructive Sleep Apnea Syndrome

- Moderate diffuse hepatic disease

- Multinodular goiter with thyroidectomy for approximately 6 years

Medication:

Sodium levothyroxine; Furosemide; Clopidogrel; Carvedilol; Trazodone; Esomeprazole; Diazepam; Metformin; Calcitriol.

Recent screening tests:

Colonoscopy: colonic diverticulitis + internal hemorrhoids grade II. Perform biopsy to check for microscopic colitis
Triglicéridos: 104,0mg/dL $(<150,0)$

Alanina Aminotransferase: 26,4U/L $(14,0$ - 54,0)

Aspartato Aminotransferase: 25,2U/L $(10,0-35,0)$

Gamaglutamiltransferase: 41,0U/L $(7,0-50,0)$

Proteína C reativa: $17,14 \mathrm{mg} / \mathrm{L}(<6,10)$

Hormonologia

T4 livre:1,4ng/dL (30,0 - 260)

TSH:5,32 $\mu \mathrm{UI} / \mathrm{mL}(0,30-4,70)$

Imunologia

IgA (soro): 494,0mg/dL $(70,0-400,0)$

Factor reumatoide: $<12,5 \mathrm{UI} / \mathrm{Ml}(<16,0)$

Anticorpos Anti-Nucleares: negativo

Ac. Anti-Citoplasma Neutrófilo, IgG: negativo

Ac. Anti-CCP, IgG: 0,0UA/mL $(<5,0)$

Ac. Anti-Transglutaminase Tecidular, IgA: 2,2U/mL (negativo: $<10$ )

Urina

Creatinina: $93,0 \mathrm{mg} / \mathrm{dL}(30,0-1,7)$

Microalbuminúria: $<0,7 \mathrm{mg} / \mathrm{dL}(<2,0)$

Avaliação clínica:

Antecedentes pessoais:

- Obesidade II

- Síndrome depressivo

- Hipertensão arterial

- Doença valvular cardíaca - esclerose ligeira da válvula aórtica; ligeira hipertrofia ventricular esquerda; discreta regurgitação mitral e tricúspide

- Diabetes mellitus tipo 2

- Fibromioma do útero

- Fibromialgia

- Síndrome da Apneia Obstrutiva do Sono

- Esteatose hepática difusa moderada

- Bócio multinodular com tiroidectomia há cerca de 6 anos.

Medicação:

Levotiroxina sódica; Furosemida; Clopidogrel; Carvedilol; Trazodona; Esomeprazol; Diazepam; Metformina; Calcitriol.

Exames complementares de diagnóstico recentes:

Colonoscopia: Diverticulite cólica + Hemorroidas internas grau II. Faz biopsia para despiste de colite microscópica 
Histology: Changes compatible with lymphocytic colitis

Intestinal transit: 4 to 5 pasty periods per day, without blood or mucus.

Eating habits:

Wake up at 7 a.m.

Breakfast: 7:15 a.m.

3 white bread toasts +1 cup of coffee with half-fat milk

Morning snack: 10:30 a.m.

1 natural yogurt +1 banana +1 water and salt wafer

Lunch: 1:30 p.m.

Dish: 2 tablespoons of white rice + meat or fish $(100 \mathrm{~g})$

+ vegetable $(50 \mathrm{~g})$

Dessert: 1 piece of fruit (assorted)

Afternoon snack: 17 p.m.

1 white bread with 1 slice of cheese +1 cup of coffee with half-fat milk

Dinner: 8 p.m.

Chicken broth / meat

1 piece of fruit (varied)

Supper: 22 p.m.

1 natural yogurt +2 water and salt biscuits

Lie down at 11:30 p.m.

Water / tea consumption: 5 cups per day

Environment, behavior and social

She lives with her 69-year-old husband, also retired.

Due to her clinical situation, and having episodes of diarrhea, she is afraid to leave home, having little social contact.

Has a sedentary activity.

Questions:

1. Lymphocytic colitis or microscopic colitis?

2. What is the main symptom?

3 . Why colon biopsies are required to definitively distinguish lymphocitic colitis from the much more common irritable bowel syndrome?

4. What medical condition should have been excluded
Histologia: Alterações compatíveis com colite linfocítica

Trânsito intestinal: 4 a 5 dejeções pastosas por dia, sem sangue ou muco.

Hábitos alimentares:

Acorda às $7 \mathrm{~h}$

Pequeno-almoço: $7 \mathrm{~h} 15$

3 torras de pão branco +1 chávena de café com leite meio gordo

Lanche da manhã: $10 \mathrm{~h} 30$

1 iogurte natural +1 banana +1 bolacha de água e sal

Almoço: $13 \mathrm{~h} 30$

Prato: 2 colheres de sopa de arroz branco + carne ou peixe $(100 \mathrm{~g})+$ verdura $(50 \mathrm{~g})$

Sobremesa: 1 peça de fruta (variada)

Lanche da tarde: $17 \mathrm{~h}$

1 pão branco com 1 fatia de queijo +1 chávena de café com leite meio gordo

Jantar: $20 \mathrm{~h}$

Caldo de galinha/carne

1 peça de fruta (variada)

Ceia: $22 \mathrm{~h}$

1 iogurte natural +2 bolachas de água e sal

Deita-se às $23 \mathrm{~h} 30$

Consumo de água/chá: 5 chávenas por dia

Ambiente, comportamento e social:

Vive com o marido de 69 anos, também reformado.

Face à sua situação clínica, e pelo facto de estar com episódios de diarreia, tem receio de sair de casa, tendo pouco contacto social.

Apresenta uma atividade sedentária.

Questões:

1. Colite linfocítica ou colite microscópica?

2. Qual é o principal sintoma?

3. Porque são necessárias biópsias ao cólon para distinguir definitivamente a colite linfocítica da síndrome do intestino irritável, que é mais comum?

4. Qual é a patologia que deve ser excluída quando 
when presented a patient with lymphocytic colitis and why?

5. No curative therapy currently exists for lymphocytic colitis. What should be the main goals treatment? um paciente apresenta sintomas de colite linfocítica e porquê?

5. Atualmente não existe terapia curativa para a colite linfocítica. Qual deve ser o principal objetivo de tratamento?

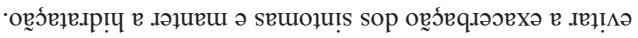

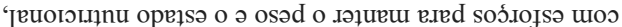

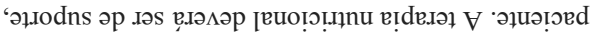

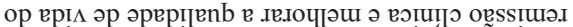

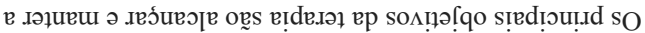

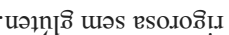

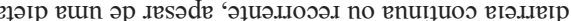

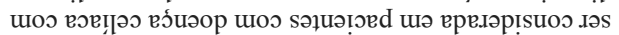

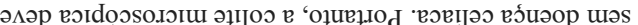

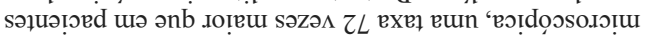

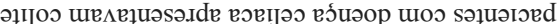

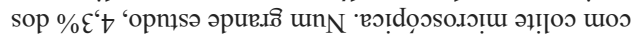

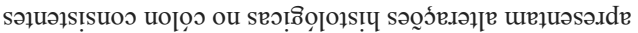

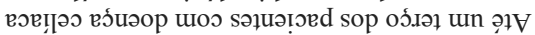

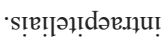

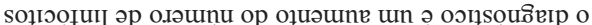

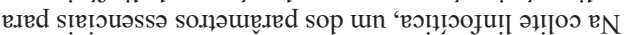

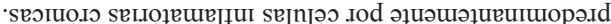

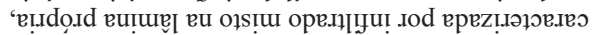

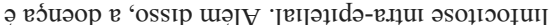

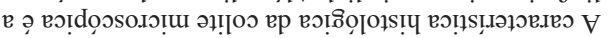

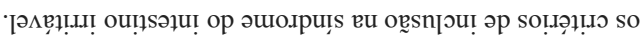

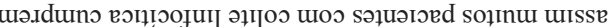

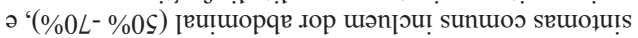

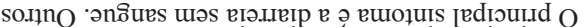

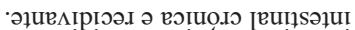

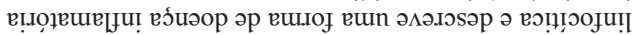

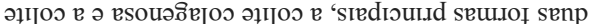

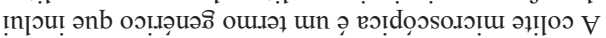

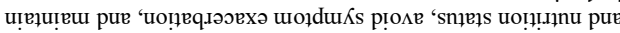

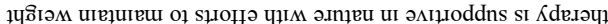

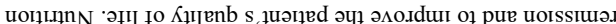

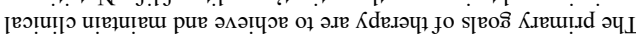

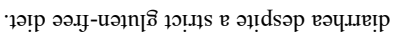

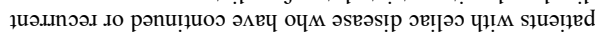

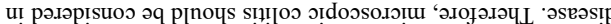

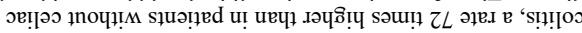

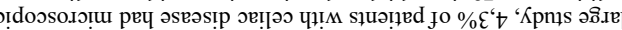
әио UI 'sı!

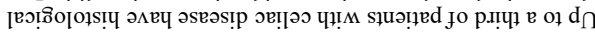

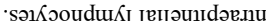

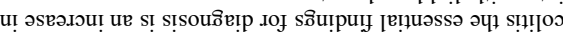

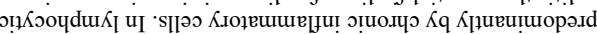

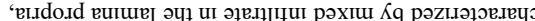

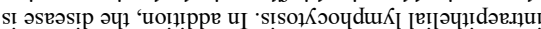

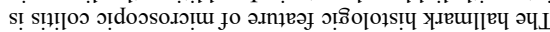

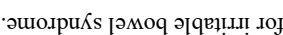

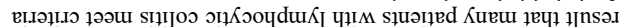

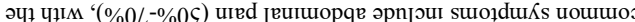

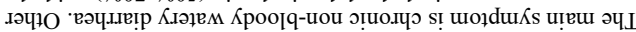

$$
\text { •วs.snos }
$$

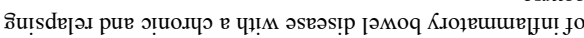

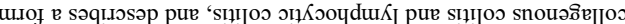

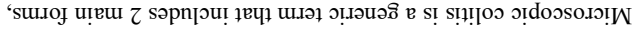




\section{References/ Referências}

1. Bouma G. Munch A. Microscopic colitis. Dig Dis 2015;33(2):208-14.

2. Chande N, Al Yatama N, Bhanji T, Nguyen TM, McDonald JW, MacDonald JK. Interventions for treating lymphocytic colitis. Cochrane Database Syst rev 2017 Jul 13;7:CD006096.

3. - Clara APMS, Magnago FD, Ferreira JN, Grillo TG. Microscopic colitis: A literature review. Rev Assoc Med Bras 2016;62(9):895-900.

4. Farrukh A, Mayberry JF. Microscopic colitis: a review. Coloretal Dis 2014 dec;16(12):957-64.

5. Jauregui-Amezaga A, Vermeire S, Geboes K. Contemporary methods for the diagnosis and treatment of microscopic colitis. Expert Rev Gastroenterol Hepatol 2016;10(1):47-61.

6. Kamp Ej, Kane JS, Ford AC. Irritable Bowel Syndrome and Microscopic Colitis: A Systematic Review and Meta-analysis. Clin Gastroenterol Hepatol 2016 May; 14(5):659-68.

7. Mullhaupt B, Guller U, Anabiarte M, Guller R, Fried M. Lymphocytic colitis: clinical presentation and long term course. Gut 1998;43:629-33.

8. Okamoto R, Negi M, Tomii S, Eishi Y, Watanabe M. Diagnosis and treatment of microscopic colitis. Clin J Gastroenterol 2016;9:169-74.

9. Pardi DS. Microscopic colitis. Clin Geriatr Med 2014 feb;30(1):55-65. 